\title{
Mecanismos moleculares de ação anti-inflamatória e antioxidante de polifenóis de uvas e vinho tinto na aterosclerose
}

SÉFORA-SOUSA, M. ${ }^{1 *}$; DE ANGELIS-PEREIRA, M.C. ${ }^{2}$

${ }^{1}$ Universidade de São Paulo - USP, Avenida Dr. Arnaldo, 715, CEP. 01246 - 904, São Paulo-SP, Brasil.

${ }^{2}$ Universidade Federal de Lavras - UFLA, Campus Universitário, Caixa Postal 3037, CEP. 37200 - 000, Lavras

- Minas Gerais, Brasil. *E-mail: marisefora@usp.br

RESUMO: Este trabalho visa investigar os possíveis mecanismos moleculares de ação dos polifenóis de uvas e vinho tinto contra os processos oxidativos e inflamatórios envolvidos na aterosclerose. Trata-se de um estudo de revisão de literatura realizado por meio de buscas nas bases de dados PubMed e ScienceDirect utilizando os seguintes descritores: vinho tinto, uva, polifenóis, resveratrol, aterosclerose, LDL-oxidada, inflamação, estresse oxidativo, NF-kB, AP-1, Nrf2, SIRT-1, MicroRNA. Embora tais mecanismos ainda não estejam totalmente esclarecidos, os estudos indicam que compostos fenólicos presentes em uvas podem se complexar com metais (como ferro e cobre) que provocam peroxidação dos lipídios, aumentam a atividade antioxidante do plasma, associam-se com a LDL-c aumentando sua resistência à oxidação, preservam a atividade da enzima paraoxonase, neutralizam radicais livres, e ativam fatores de transcrição como o Nrf2, que aumenta a expressão de genes que codificam proteínas importantes na defesa antioxidante, como a superóxido dismutase e a glutationa peroxidase. Além disso, os polifenóis inibem a fosforilação de MAP quinases, inibindo assim os fatores de transcrição NF-kB e AP-1 e, consequentemente, reduzindo a síntese do TNF-á, interleucinas, moléculas de adesão e quimiocinas. Também inibem a atividade das enzimas cicloxigenase e lipoxigenase. Outro mecanismo proposto é a ação do resveratrol sobre a atividade de deacetilases de histonas, como a SITR-1. Estas ações em conjunto reduzem a oxidação da LDL-c e o processo inflamatório, atenuando o processo aterogênico.

Palavras-chave: Resveratrol, LDL-c, Nrf2, NF-kB, AP-1, aterosclerose.

ABSTRACT: Molecular mechanisms of action of grape and red wine polyphenols against oxidative and inflammatory processes in atherosclerosis. This study aimed to investigate the possible molecular mechanisms of action of grape and red wine polyphenols against oxidative and inflammatory processes in atherosclerosis. This is a literature review performed by searching in the PubMed and ScienceDirect databases using the following descriptors: red wine, grape, polyphenols, resveratrol, atherosclerosis, oxidized LDL, inflammation, oxidative stress, NF-kB, AP-1, Nrf2, SIRT-1, MicroRNA. Although the mechanisms are not fully understood, studies indicate that phenolic compounds possibly complex with metals (such as iron and copper), or else they increase the antioxidant activity of plasma and also preserve the activity of enzyme paraoxonase. Moreover, the polyphenols neutralize free radicals and activate transcription factors, for example Nrf2, which increases the expression of genes that encode important proteins in antioxidant defense (such as superoxide dismutase and glutathione peroxidase). Additionally, polyphenols inhibit the phosphorylation of MAP kinases, causing a blocking effect on the transcription factors NF-kB and AP-1 and, consequently, blocking the synthesis of TNF- $\alpha$, interleukins, chemokines and molecules adhesion. Moreover, it can inhibit the activity of the cycloxygenase and lipoxygenase enzymes. Another proposed mechanism is the action of resveratrol on the activity of histone deacetylases, as SIRT-1. These actions together reduce the oxidation of LDL-C and the inflammatory process, attenuating the atherogenic process.

Keywords: Resveratrol, LDL-c, Nrf2, NF-kB, AP-1, atherosclerosis.

Recebido para publicação em 28/05/2012

Aceito para publicação em 20/02/2013

Rev. Bras. PI. Med., Campinas, v.15, n.4, p.617-626, 2013. 


\section{INTRODUÇÃO}

A aterosclerose, a qual se caracteriza pelo estreitamento do calibre interno dos vasos sanguíneos, está relacionada com diversos fatores etiológicos, entre eles a hipercolesterolemia, especialmente aumento da lipoproteína de baixa densidade (LDL-c) (Mehta et al., 2006). Associado a isso, pesquisas demonstram que a oxidação da LDL-c e a secreção de mediadores inflamatórios são elementos-chave na iniciação e progressão da aterosclerose (Mclaren et al., 2010).

O interesse em estudar a relação entre uvas e vinho tinto e as doenças cardiovasculares (DCV) surgiu há muitos anos, quando se observou que a população francesa, apesar do elevado consumo de gordura saturada, apresentava baixos índices de doenças coronarianas, o que ficou conhecido como "paradoxo francês" (Renaud \& Lorgeril, 1992). Desde então, vários estudos epidemiológicos têm mostrado associação inversa entre o consumo de polifenóis (principalmente o resveratrol), presentes não só no vinho, mas também em suco de uva e na pele e sementes da fruta, e a incidência de DCV (Manach et al., 2005; Vita, 2005).

O resveratrol $\left(3,5,4^{\prime}\right.$ trihidroxiestilbeno) é uma fitoalexina que foi isolada pela primeira vez em 1940 de raízes de Veratrum grandiflorum O. Loes e, posteriormente, em 1963, de raízes de Polygonum cuspidatum (Baur \& Sinclair, 2006), mas que atraiu pouco interesse dos pesquisadores. Até que, em 1992, Siemann e Creasy reportaram que o resveratrol seria o responsável pelos efeitos cardioprotetores do vinho tinto. Desde então, inúmeros trabalhos têm reportado que o resveratrol pode prevenir ou diminuir a progressão de hipertensão, aterosclerose, obesidade, diabetes, câncer, doença de Alzheimer, entre outras (Xu \& Si, 2012).

Este polifenol é um estilbeno formado através de uma reação de condensação entre 3 moléculas de malonil- CoA e uma molécula de 4 - cumaroilCoA. Sua síntese ocorre em resposta a ambientes estressantes, como infecções microbianas, radiação ultravioleta e flutuações de temperatura. Pode ser encontrado nas configurações cis ou trans (Hao \& $\mathrm{He}, 2004)$. Além de reveratrol, uvas (Vitis vinifera, Vitis rotundifolia e Vitis labrusca) e vinho tinto também são fontes de proantocianidinas, ácido elágico, campferol, miricetina, quercetina, malvidina, peonidina, cianidina e catequina (Xia et al., 2010).

Os polifenóis, incluindo o resveratrol, têm mostrado elevado efeito cardioprotetor possivelmente pela habilidade em reduzir o colesterol total e LDL-C, inibir a agregação plaquetária, estimular a vasodilatação e enzimas antioxidantes, bem como inibir vias pró-inflamatórias (Rahman et al., 2008). No entanto, os mecanismos de ação destes polifenóis ainda são controversos e não estão totalmente esclarecidos. Assim, esta revisão busca investigar os possíveis mecanismos moleculares de ação dos polifenóis de uvas e vinho tinto contra os processos oxidativos e inflamatórios envolvidos na aterosclerose.

\section{MÉTODOS}

Trata-se de um estudo de revisão de literatura realizado por meio de buscas nas bases de dados PubMed e ScienceDirect utilizando os seguintes descritores: vinho tinto, uva, polifenóis, resveratrol, aterosclerose, LDL-oxidada, inflamação, estresse oxidativo, NF-kB, AP-1, Nrf2, SIRT-1, MicroRNA, no idioma correspondente ao do banco de dados consultado e agrupadas de maneiras diversas para otimizar a busca.

Os critérios de seleção foram artigos publicados nos últimos doze anos, incluindo ensaios originais e revisões, que investigaram os processos oxidativos e inflamatórios envolvidos na aterosclerose e estudos que avaliaram os efeitos dos polifenóis, especialmente o resveratrol, sobre alvos genéticos importantes no estresse oxidativo e na inflamação. Além disso, os artigos foram selecionados quanto à originalidade e à relevância, considerando-se o rigor e a adequação do delineamento experimental. Também foram incluídos trabalhos clássicos relacionados à temática.

Dos 250 trabalhos pesquisados, selecionouse 75 artigos, sendo 58 trabalhos da base PubMed e 17 trabalhos da ScienceDirect.

\section{Processos Oxidativos e Inflamatórios Envolvidos na Aterosclerose}

A aterosclerose é caracterizada pelo estreitamento ou oclusão da artéria por um ateroma, o qual é constituído por diversos elementos, como lipídios, elementos fibrosos, plaquetas, monócitos e cálcio. Neste caso, as lesões iniciais do processo aterosclerótico podem ser provocadas por diversos agentes e condições fisiopatológicas, como uso de tabaco, hipertensão arterial, obesidade, diabetes mellitus tipo 2, excesso de colesterol total, baixos níveis de lipoproteína de elevada densidade (HDL-c), e, especialmente, elevados níveis de LDL-c, a qual pode se acumular na camada íntima da artéria (Luz \& Coimbra, 2004).

Segundo a hipótese oxidativa, a LDL-c nativa, ao penetrar na íntima do vaso, sofre oxidação, a qual pode ocorrer pela ação de mieloperoxidases, lipoxigenases e espécies reativas de oxigênio (ERO), como superóxido e peróxido de hidrogênio. A LDL-c oxidada é citotóxica, gerando lesões nas células endoteliais (Steinberg et al., 1989; Hansson \& Hermansson, 2011; Rocha \& Libby, 
2009). Neste processo, em resposta à agressão, ocorre a produção de moléculas de adesão para leucócitos/monócitos [como molécula de adesão intercelular (ICAM-1), e-selectina e molécula de adesão vascular (VCAM-1)], as quais promovem o rolamento e a adesão destas células no vaso. Uma vez aderidos, os monócitos migram para a camada íntima da artéria em resposta a estímulos quimioatraentes, especialmente a MCP-1 (proteína quimiotática para monócitos-1), onde se diferenciam em macrófagos (Hansson \& Libby, 2006; Agrawal et al., 2010). Posteriormente, a LDL-c oxidada é reconhecida por receptores scavengers localizados nos macrógafos, os quais, ao internalizarem esta lipoproteína, formam as células espumosas (células gordurosas que originarão o ateroma) (Brown \& Goldstein, 1983; Rocha \& Libby, 2009). Ressalta-se que os receptores da LDL-c oxidada não apresentam feedback em resposta ao excesso de colesterol celular, o que leva a um excessivo acúmulo de gordura (Steinberg \& Witztum, 2010).

Tanto a LDL-c oxidada como os produtos dessa oxidação podem se ligar aos receptores scavengers e aos receptores toll-like (TLR) localizados nos macrófagos. A internalização de LDL-c oxidada pelos receptores scavengers ativa os inflamossomas, estimulando a secreção de IL-1ß; enquanto que a ativação de TLR desencadeia a via do NF-карра B (NF-kB) e da proteína ativadora-1 (AP-1), importantes fatores de transcrição que modulam a expressão de moléculas pró-inflamatórias (Hansson \& Hermansson, 2011). $\mathrm{Na}$ figura 1 tem-se um resumo esquemático do processo de oxidação da LDL-c e seus efeitos na aterosclerose.

A proteína ativadora-1 (AP-1) encontrase frequentemente como um heterodímero de c-Jun e c-Fos, tendo sua ativação regulada pelas proteínas quinases ativadas por mitógenos (MAPK). As MAPK englobam um grande número de quinases que fosforilam serina e treonina e regulam processos celulares, como crescimento, proliferação e diferenciação celular. Dividem-se em três subfamílias: quinases reguladas por sinal extracelular (ERK); quinases c-Jun N-terminal (JNK) e as quinases p38 (Martindale \& Holbrook, 2002). Tipicamente, a ativação das MAPK é iniciada por citocinas inflamatórias, como o TNF-a, que ao interagirem com seus receptores, levam à ativação de uma cascata de fosforilações. A fosforilação da JNK e p38 requer a atividade de MAPK quinases upstream (MAPKKs, por exemplo, MKK3 e MKK6) e MAPK quinase quinases [MAPKKKs - por exemplo, quinase reguladora de sinais apoptóticos-1 (ASK1) e TGF- $\beta$ quinase ativada-1 (TAK-1)]. As formas fosforiladas da JNK e p38 ativam fatores de transcrição pertencentes à proteína ativadora-1
(AP-1), incluindo c-Jun e fator de ativação 2 de transcrição (ATF-2), os quais ativam a transcrição de genes que codificam moléculas de adesão como VCAM-1 e citocinas inflamatórias (Warboys et al., 2011). Este processo de ativação está esquematizado na Figura 2.

A ativação da via NF-kB tem um papel central na inflamação, através da regulação de genes que codificam citocinas pró-inflamatórias, moléculas de adesão, quimiocinas, fatores de crescimento, enzima cicloxigenase-2 (COX2) e enzima óxido nítrico sintase induzível (iNOS). O NFkB é um dímero de proteínas, que inclui a p50 e p65, encontrado em sua forma inativa (ligado ao inibidor $\mathrm{Kb}(\mathrm{I} \mathrm{BB} \alpha / \beta))$ no citoplasma das células. Citocinas pró-inflamatórias, como o TNF-a, lipopolissacarídeos bacterianos (LPS) e a LDL-c oxidada, ao interagirem com seus respectivos receptores ativam proteínas quinases, como a IkB quinase/IKK (um complexo protéico composto por duas quinases IKK1/IKKa e IKK2/IKK $\beta$ ) ou MAPK. A IKK ou MAPK fosforilam - $I k B a / \beta$, o qual é ubiquitinado e degradado no proteassoma, liberando o NF-kB para interagir com região a promotora do DNA e estimular a expressão de genes que codificam proteínas inflamatórias (Figura 2). Por ser redox-sensível este fator de transcrição pode ser ativado por espécies reativas de oxigênio (EROS) (Robbesyn et al., 2004; Kundu \& Surh, 2004; Tedgui \& Mallat, 2006).

Associado a isso, pesquisas indicam que o fator nuclear eritróide (Nrf2), um relavante fator de transcrição que ativa genes codificantes de proteínas antioxidantes, é ativado em condições de estresse intenso e pode ser um agente protetor das artérias via regulação negativa das MAPK, reduzindo, por exemplo, a sinalização da MKK3/6 e, consequentemente, a ativação de fatores de transcrição pró-inflamatórios (Warboys et al., 2011). Estes achados apontam para tentativa do organismo em recuperar a homeostasia frente a situações de estresse como acontece na formação da placa aterosclerótica.

A resposta inflamatória envolvida na aterosclerose desencadeia não só a liberação de citocinas e quimiocinas, mas também de proteínas da fase aguda, principalmente proteína $C$ reativa (PCR). Vários estudos mostram associação entre os níveis elevados de PCR e a progressão da aterosclerose, provavelmente, devido à capacidade desta proteína em aumentar a expressão de MCP-1 e de moléculas de adesão, favorecendo a ligação dos monócitos às células endoteliais e por mediarem a captação de LDL-c oxidada pelos macrófagos, promovendo a formação de células espumosas (Libby et al., 2002; Devaraj et al., 2003). Kaur et al.(2007) demonstraram in vitro que a IL-6 e a IL-1 $\beta$ ativam a fosforilação da p38 e da p44/42, as quais sinalizam o aumento da expressão da PCR. 


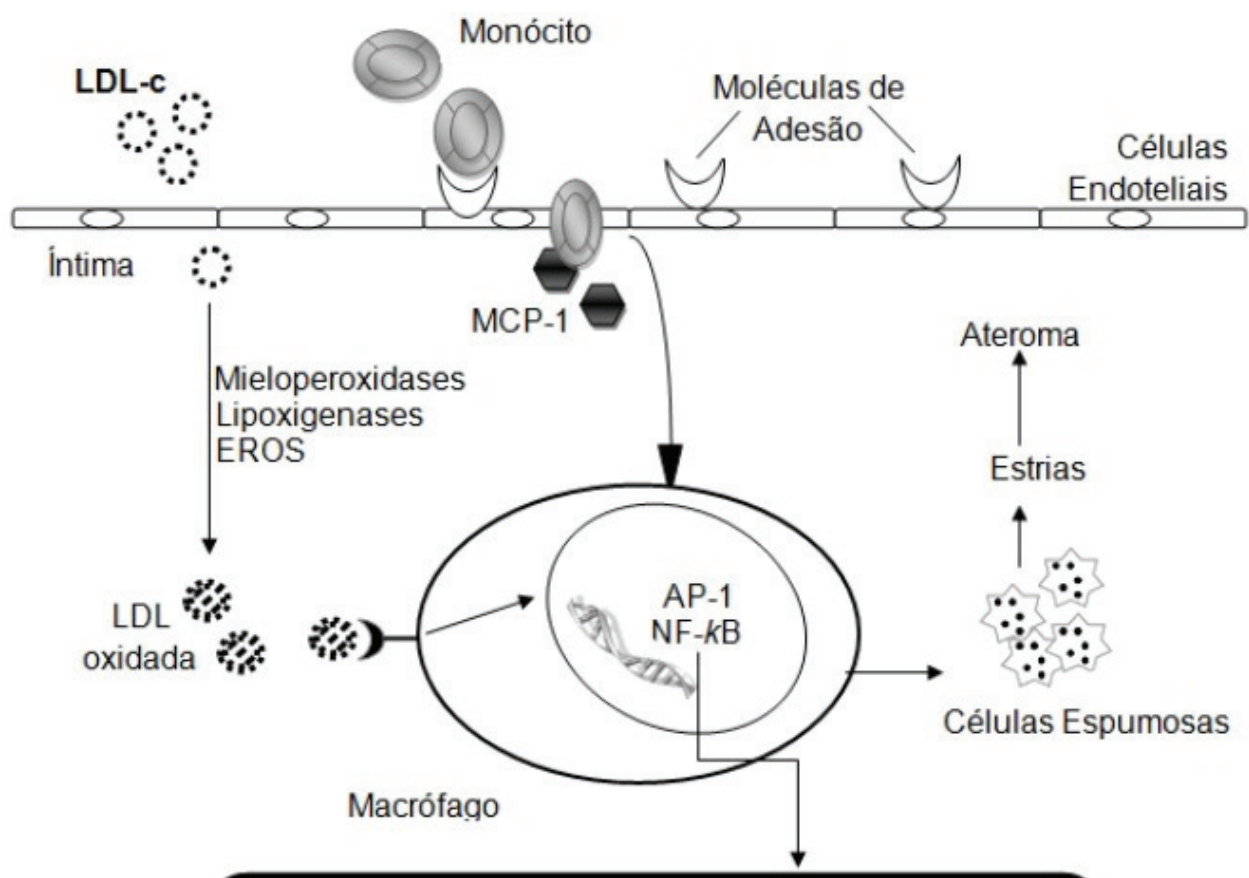

Estímulo à síntese de moléculas de adesão, EROS,

eicosanóides, citocinas pró-inflamatórias e quimiocinas.

FIGURA 1. Internalização da LDL-c oxidada por receptores scavengers e aterosclerose.

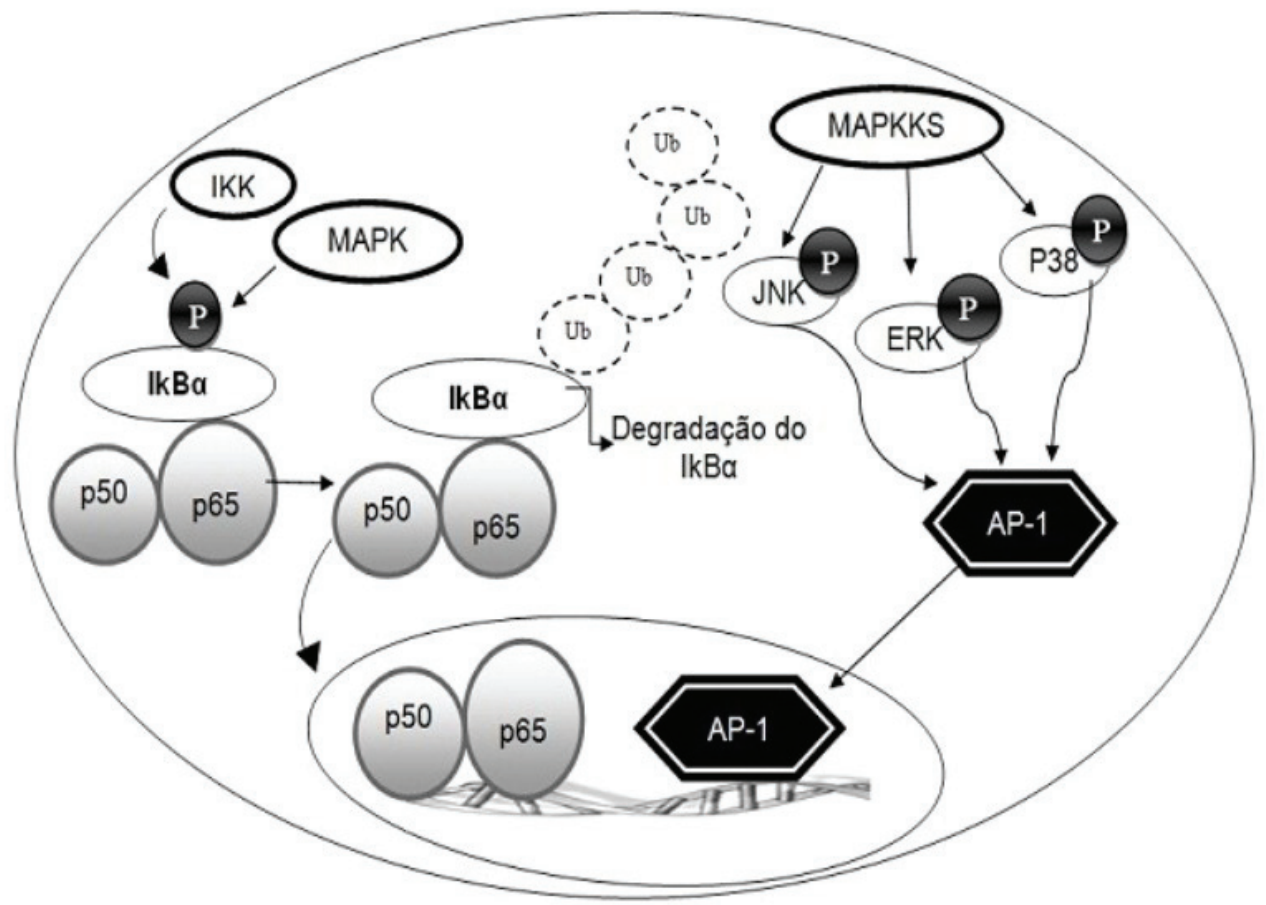

FIGURA 2. Processo de ativação dos fatores de transcrição NF-kappaB e AP-1. 


\section{Mecanismos moleculares de ação de polifenóis de uvas e vinho tinto contra os processos oxidativos}

$\mathrm{Na}$ última década, estudos vêm sendo conduzidos com o intuito de verificar as propriedades antioxidantes de uvas e do vinho tinto. Experimentos in vitro, em animais e em humanos, vêm mostrando que o consumo desses produtos pode elevar a capacidade antioxidante do plasma e reduzir a susceptilidade da LDL-c à oxidação.

Já foi demonstrado que os polifenóis (catequina, epicatequina, quercetina e resveratrol) presentes no vinho tinto podem atuar diretamente contra as espécies reativas de oxigênio (ERO) e nitrogênio (ERN) em macrófagos de murinos (Č́ž et al., 2008). Em aortas de ratos, a suplementação com resveratrol (50 mg /L na água de beber durante 21 dias) provocou inibição da NADPH-oxidase e diminuição na produção de superóxido (Soylemez et al., 2008; 2009). Associada à atividade scavenger, polifenóis podem quelar metais, como ferro e cobre, e assim inibir a formação de radicais livres por meio da reação de Haber-Weiss/Fenton (Rodrigo et al., 2011).

Por outro lado, Urpí-Sardà et al. (2005) constataram a presença de metabólitos do resveratrol (trans-resveratrol-3-O-glicuronídeo, cis-resveratrol3-O glucuronideo, cis-resveratrol-3-O-glicosídeo, bem como trans-resveratrol livre) na LDL-C de homens saudáveis $(n=11)$, sugerindo que estes compostos podem transferir o seu efeito antioxidante a esta lipoproteína. Já em camundongos deficientes em Apo E, o consumo de vinho tinto, durante dois meses, resultou em diminuição da oxidação da LDL-c em $40 \%$, redução do tamanho das lesões ateroscleróticas e menor número das células espumosas. Além disso, constatou-se aumento da atividade da paraoxonase sérica (Aviram \& Fuhrman, 2002).

De forma semelhante, em hamsters alimentados com dieta aterogênica, a suplementação com 7,14 mL. $\mathrm{kg}^{-1}$ de vinho merlot (sem álcool) por 12 semanas também reduziu a produção de superóxido e melhorou a atividade da enzima paraoxonase sérica (Suh et al., 2011). Em mulheres pré e pósmenopausa o consumo de $36 \mathrm{~g}$ de liofilizado de uva Vitis vinifera L., por quatro semanas, reduziu os níveis de F2-isoprostanos (Zern et al., 2005).

Extratos de diferentes variedades de uva (Cannonau, Vermentino e Malvasia) também foram capazes de reverter os efeitos do oxidante tert-butil hidroperóxido (TBH) em enterócitos humanos, reduzindo a produção de malondialdeído (MDA), hidroperóxidos e a oxidação do colesterol (Deiana et al., 2012)

Em um estudo randomizado, cross-over, a ingestão de vinho tinto sem álcool (300 $\mathrm{ml} / \mathrm{dia})$, por uma semana, resultou em aumento da capacidade antioxidante do plasma e maior atividade das enzimas glutationa redutase, catalase e superóxido dismutase (SOD) em indivíduos saudáveis, embora não tenham sido verificadas alterações em alguns antioxidantes endógenos (ácido úrico, bilirrubina, albumina) (Noguer et al., 2012).

Em cardiomiócitos exposto à injúria celular, o pré-tratamento com resveratrol $(30 \mu \mathrm{M})$ melhorou a atividade das enzimas catalase e superóxido dismutase. E em ratos hipertensos, a suplementação com resveratrol $(2,5 \mathrm{mg} / \mathrm{kg} / 10$ semanas) preveniu o aumento da produção de peróxido de hidrogênio (Movahed et al., 2012). Além disso, já foi verificado que proantocianidinas de uvas inibem a atividade da NADPH oxidase com consequente redução de ERO em céluas endoteliais (Álvarez et al., 2012).

A análise proteômica de células endoteliais humanas tratadas com resveratrol revelou ainda alterações pós-transcricionais em várias proteínas, entre elas a nicotinamida-nucleotídeo pirofosforilase, uma enzima-chave na síntese de novo de $\mathrm{NAD}^{+}$que é um cofator essencial para o equilíbrio redox das células (Shao et al., 2012).

Baseado nestes resultados pode-se inferir que os polifenóis de uvas e vinho tinto previnem ou reduzem o estresse oxidativo por meio da atividade scavenger de radicais livres, complexação com metais oxidantes, associação com a LDL-c aumentando sua resistência à oxidação, aumento de antioxidantes endógenos e por meio da modulação da atividade de enzimas-chave na defesa antioxidante.

A modulação das enzimas antioxidantes por polifenóis parece ocorrer via ativação do fator de transcrição "Nuclear factor-E2-related factor" (Nrf2) (Chuang \& Mcintosh, 2011; Palsamy \& Subramanian, 2011). Este pode ser um mecanismo crucial pelo qual os polifenóis conferem cardioproteção.

Em condições basais, o Nrf2 encontra-se no citoplasma ligado ao complexo repressor Kelchlike $\mathrm{ECH}$-associated protein 1 (KEAP-1), o qual promove ubiquitinação e degradação do Nrf2 no proteasoma. No entanto, em resposta ao estresse oxidativo ou eletrofílico, o Nrf2 dissocia-se do KEAP-1 e transloca-se para o núcleo onde se liga aos elementos de resposta antioxidante (ARE) ou elementos de resposta à eletrófilo (EpRE), ativando a transcrição de enzimas antioxidantes/detoxificantes (Chapple et al., 2012; Takaya et al., 2012). Em adição, o Nrf2 inibe a expressão de mediadores pró-inflamatórios por meio da regulação de enzimas anti-inflamatórias, como a heme oxigenase-1(HO-1) (Kim et al., 2010).

Neste contexto, observou-se em células endoteliais que o resveratrol aumentou

Rev. Bras. PI. Med., Campinas, v.15, n.4, p.617-626, 2013. 
significativamente a atividade transcricional do Nrf2, o qual esteve associado ao incremento da expressão dos genes relacionados à $N A D(P)$ $\mathrm{H}$ :quinona oxidorredutase-1, $y$-glutamil cisteina sintetase e HO-1 (Ungvari et al., 2010). Em ratos, a suplementação com resveratrol $(10 \mathrm{mg} / \mathrm{kg} / \mathrm{dia})$ reduziu os níveis hepáticos de dienos conjugados e aumentou a atividade das enzimas catalase e SOD. Essa atenuação do estresse oxidativo hepático também foi correlacionada com o incremento nos níveis de Nrf2 (Bagul et al., 2012).

Ademais, o Nrf2 pode ser ativado por diversas vias de sinalização, incluindo ativação de ERK2, JNK1, proteína quinase-C (PKC) e fosfatidilinositol 3-quinase (PI3K) (Lee et al., 2012). No estudo de Bak et al. (2012), proantocianidinas de sementes de Vitis amurensis incrementaram a atividade de enzimas antioxidantes mediada pelo Nrf2/ARE via ativação das vias p38 e PI3K/Akt em hepatocarcinoma. Em concordância com este estudo, Rodríguez-Ramiro et al. (2012) mostraram que a proantocianidina B2 induz a expressão de glutationa S-transferase P1 e a translocação do Nrf2 para o núcleo. Interessantemente, as quinases ERKs e p38 também foram ativadas.

Zhang et al. (2013) sugerem ainda que o aumento da atividade da SOD pelo resveratrol pode ocorrer via ativação da sirtruina-1 (SIRT1), uma deacetilase de histona dependente de $\mathrm{NAD}^{+}$. Segundo Yao \& Rahman (2012), SIRT-1 protege contra o estresse oxidativo por elevar a atividade transcricional das proteínas Forkhead Box (FOXO3), as quais regulam a expressão de genes antioxidantes, como os da catalase e SOD.

Algumas pesquisas também aventam a possibilidade dos polifenóis do vinho tinto atuarem sobre o fator de crescimento de endotélio vascular (VEGF), um potente mediador da angiogênese que participa da coagulação do fibrinogênio e deposição de gel de fibrina no crescimento de novos vasos sanguíneos. Fatores de crescimento, como o $\mathrm{PDGF}_{\mathrm{AB}}$, promovem a ação da NADPH oxidase, levando a geração de ERO, os quais ativam proteínas quinases ativadores de mitógenos (MAPK), como a p38. Esta, por sua vez, ativa o fator induzido por hipóxia (HIF-1), o qual promove aumento na expressão do fator de crescimento endotelial vascular (VEGF) nas células musculares lisas dos vasos. Compostos polifenólicos do vinho tinto, ao inibirem a produção de radicais livres, inibiriam a fosforilação de MAPK p38 e, assim, diminuiriam a expressão do VEGF (Oak et al., 2005).

Embora os produtos da uva apresentem atividade antioxidante, essa propriedade é dosedependente e, portanto, concentrações elevadas dos compostos fenólicos podem apresentar efeito pró-oxidante, levando a danos celulares e a modificações oxidativas no DNA (Lastra \& Villegas, 2007). Entretanto, algumas pesquisas têm verificado que a ação pró-oxidante de polifenóis como o resveratrol pode ser um mecanismo natural e importante para ativar fatores promotores de vasodilatação.

Nesse sentido, Ndiaye et al (2003) buscando investigar os mecanismos envolvidos na ação do vinho como agente relaxante, observaram aumento do radical superóxido nas artérias coronárias suínas tratadas com os polifenóis do vinho tinto. Segundo os autores, a produção deste radical está relacionada com a formação do fator hiperpolarizante dependente de endotélio (EDHF), o qual conduz à hiperpolarização, fenômeno correlato ao relaxamento. Ou seja, os polifenóis atuam como vasodilatadores das artérias coronárias por estimularem a formação de EDHF endotelial, o que ocorre por meio de um mecanismo redoxsensível (dependente de superóxido). Considerando os polifenóis como potentes agentes varredores de radicais livres, os autores acreditam que, provavelmente, a geração de superóxido pelos polifenóis seja realizada localmente e de forma controlada para ativar vias de transdução envolvidas na hiperpolarização da membrana ou para afetar diretamente a atividade de canais de potássio nas células endoteliais, os quais medeiam a ação do EDHF.

\section{Mecanismos moleculares de ação de polifenóis de uvas e vinho tinto contra os processos inflamatórios}

Estudos indicam que o consumo de vinho tinto e de suco de uva está relacionado com a redução nos níveis de marcadores inflamatórios, como as citocinas e quimiocinas. Neste contexto, observou-se que a ingestão moderada de vinho tinto siciliano (250ml/dia, por 4 semanas) por homens e mulheres saudáveis $(n=48)$ causou a redução nos níveis de LDL-c oxidada, fibrinogênio, PCR e aumentou significativamente as concentrações de HDL-c, Apo A1 e da proteína fator beta de transformação de crescimento (TGFâ1) (Avellone et al., 2006).

Estruch et al. (2004) verificaram que o consumo do vinho tinto $(360 \mathrm{ml})$ durante 28 dias por indivíduos saudáveis $(n=40)$ resultou em diminuição nos níveis sanguíneos de fibrinogênio $(-10 \%)$, IL-1á (-21\%), VCAM-1(-17\%), ICAM-1 (-9\%) e PCR (-21\%), bem como suprimiu a expressão do antígeno 1 associado à função leucocitária (LFA-1) ("27\%), da integrina Mac-1 dos leucócitos ("27\%), do antígeno de ativação muito tardio (VLA-4) ("32\%) e MCP-1 (" $46 \%$ ). Estes dados foram confirmados por Sacanella et al. (2007) os quais também observaram redução de IL-6, e-selectina e p-selectina em 
mulheres saudáveis que consumiram vinho tinto (200 ml/ dia/ por 4 semanas).

Albers et al. (2004) investigaram o efeito do consumo de suco de uva Vitis labrusca $(7 \mathrm{ml} /$ $\mathrm{kg} / \mathrm{dia}$ ), por 14 dias, em indivíduos com doença coronariana diagnosticada $(n=20)$. Embora não tenham encontrado diferenças nos níveis de PCR e IL-8, houve redução significativa da proteína CD40L, a qual está associada ao aumento da produção de radicais livres, da expressão de moléculas de adesão e da expressão de citocinas pró-inflamatórias

Os mecanismos moleculares pelos quais os polifenóis presentes na uva e no vinho tinto atuam como anti-inflamatórios ainda não estão totalmente esclarecidos. Os estudos apontam para modulação da atividade de várias proteínas e fatores de transcrição, os quais estão envolvidos em vias metabólicas relacionadas à síntese de citocinas, quimiocinas e moléculas de adesão.

Uma das possíveis vias está relacionada com a capacidade antioxidante destes compostos, os quais, ao combaterem o estresse oxidativo (ocasionado pelo excesso de ERO) atenuam a inflamação. Isso porque as espécies reativas de oxigênio, capazes de ativar proteínas quinases (JNK, ERK e p38) e assim, aumentar a atividade de fatores de transcrição indutores da inflamação, são neutralizadas. Além disso, compostos fenólicos podem atuar diretamente sobre os fatores de transcrição envolvidos na resposta inflamatória, inibindo a expressão de citocinas (Chuang et al., 2011).

No estudo de Maggi-Capeyron et al. (2001) os ácidos fenólicos (gálico, caféico, p-cumárico, sináptico e ferúlico) presentes no vinho apresentaram, in vitro, a capacidade de modular negativamente o AP-1. De forma semelhante, o resveratrol $(50 \mathrm{mg} / \mathrm{kg} / \mathrm{dia})$ minimizou a atividade do AP-1 na retina de ratos (Kubota et al., 2010). Os polifenóis do vinho parecem modular a atividade do AP-1 por meio da inibição da PKC, da via MAPK e/ ou alterando a ligação do AP-1 ao DNA.

Gessner et al. (2012) constatatam que o efeito anti-inflamatório de extratos obtidos de farinhas de semente e de bagaço de uva também ocorre via inibição da via NF-kB. Em macrófagos humanos com inflamação mediada pelo LPS, extratos de uva (Vitis labrusca) em pó (GPE) atenuaram a fosforilação da JNK e p38 e diminuíram a degradação do IkBá e a fosforilação da c-Jun, bem como bloquearam o NF-kB. Fato interessante observado é que o GPE não produziu os mesmos efeitos na ausência do LPS (Overman et al., 2010). Em adipócitos pré-diferenciados, o GPE atenuou a inflamação mediada por TNF-á por meio da inibição da expressão das IL-6, IL-1â, IL-8, MCP-1, COX-2 e do receptor toll-like 2 e da inibição do AP-1 e do NF- $k \mathrm{~B}$, sendo o efeito dose-dependente (Chuang et al., 2011). Além disso, já foi reportado que o resveratrol pode bloquear a fosforilação da subunidade p65 do NF-kB, inibindo sua translocação para o núcleo e, consequentemente, sua ação nos genes. Este bloqueio estaria correlacionado com a inibição da fosforilação e degradação do IkBá pelo resveratrol (Cianciulli et al., 2012).

Csiszar et al. (2006) demonstraram em células endoteliais de artéria coronariana a inibição da expressão da IL-6, iNOS, ICAM-1, VCAM-1, bem como a menor ativação do NF-kB (mais de $50 \%$ ) pelo resveratrol. Em macrófagos peritoniais estimulados com lipopolissacarídeo (LPS) e interferon-ã, o tratamento com cis-resveratrol bloqueou a expressão de genes relacionados à família Rel/NF-kB/IkB, às moléculas de adesão e a proteínas da fase aguda, bem como inibiu a expressão do receptor toll-like (TLR 3) e da MCP-1 (Leiro et al., 2005). Também se observa inibição da expressão do TNF-á pelo resveratrol (Bi et al., 2005).

Ademais, já foi demonstrada in vitro a capacidade do resveratrol em inibir as enzimas lipoxigenase (Chatterjee et al., 2011)e cicloxigenase (Cianciulli et al., 2012), as quais atuam na síntese de eicosanóides a partir do ácido araquidônico. Leifert \& Abeywardena (2008) demonstraram em células Caco-2 e HepG2 a capacidade de extratos de vinho tinto e de sementes de uva em inibirem a atividade da 5-lipoxigenase. No trabalho de Yi et a. (2011), o resveratrol inibiu a expressão da COX-2 via inibição da translocação do NF-kB em macrófagos. Já Chung et al. (2011) mostraram que este estilbeno inibe in vitro a síntese de interferon-ã. Associado a isso, o resveratrol e a quercetina $(50 \mu \mathrm{M})$ inibem in vitro a expressão da PCR, em um processo não mediado pelas MAPK p38 e p44/42 (Kaur et al., 2007).

Recentemente, Noratto et al. (2011) trataram células endoteliais vasculares com inflamação induzida pelo LPS com extratos de uva Vitis rotundifolia (5-20 mg equivalente de ácido gálico/L) e mostraram que além de ocorrer inibição da tradução das interleucinas 6 e 8, mediada pela inibição da atividade do NF-kB, o extrato de uva foi capaz de induzir a expressão do microRNA 126, o qual esteve associado à diminuição da tradução da VCAM-1. Realmente, os microRNAS (moléculas de RNA não codificadores de proteínas) ao se ligarem à região 3' não traduzida de RNAs mensageiros alvo podem reduzir os níveis proteicos de seus genes-alvo e regular a sinalização celular através de alterações nos níveis de quinases e de fatores de transcrição e por modificar alvos epigenéticos (Lançon et al., 2012). Já Tili et al. (2010) verificaram up-regulation do microRNA 663, um supressor da inflamação e do fator transcricional AP-1, em monócitos tratados com resveratrol. 
Outro mecanismo proposto é a ação do resveratrol sobre a atividade de deacetilases. Nesse sentido, o resveratrol pode ativar a deacetilase de histona tipo 2 (HDAC2) ou SIRT-1, proteínas que retiram os grupos acetila das histonas do DNA, causando alteração conformacional e, desse modo, interferindo negativamente na interação dos fatores de transcrição com a região promotora do material genético (Rahman, 2008; Yun et al., 2012). A SIRT1 suprime, por exemplo, o NF-kB por deacetilação da sua subunidade RelA/p65 (Chung et al., 2010; Yao \& Rahman, 2012). Gracia-Sancho et al. (2010) demonstraram que a ativação da SIRT-1 pelo resveratrol também está envolvida na indução do fator transcricional KLF-2 (Krüppel-like factor 2) em células endoteliais humanas. Embora as ações do KLF-2 ainda não sejam claras, sua ativação tem sido implicada na modulação da angiogênese por meio do efeito vasodilatador, anti-inflamatório e antitrombótico (Atkins \& Jain, 2007).

\section{CONCLUSÕES}

Os compostos fenólicos da uva e seus derivados, como o vinho tinto, são potentes antioxidantes e desempenham papel relevante na prevenção da oxidação da LDL-c e na redução de mediadores inflamatórios, atenuando, assim, o processo aterosclerótico. Embora os mecanismos moleculares de ação não estejam totalmente esclarecidos, estudos indicam que os polifenóis modulam a atividade de diversos fatores de transcrição, principalmente do NF-kB, AP-1 e Nrf2, o que conduz à redução de citocinas inflamatórias e de moléculas de adesão e aumento da expressão de enzimas antioxidantes.

Compreender o sítio de ação destes compostos bioativos auxilia no desenvolvimento de novas estratégias de promoção de saúde e tratamento da aterosclerose, contudo, ainda há muitos processos metabólicos que precisam ser esclarecidos, o que sinaliza a necessidade de novos estudos na área, principalmente com humanos.

\section{REFERÊNCIA}

AGRAWAL, $N$. et al. Oxidation of LDL: role in Atherosclerosis. The Internet Journal of Geriatrics and Gerontology, v.6, n.1, 2010.

ALBERS, A.R. et al. The Antiinflammatory Effects of Purple Grape Juice Consumption in Subjects with Stable Coronary Artery Disease. Arterioscler. Thromb. Vasc. Biol., v.24, p.179-180, 2004.

ÁLVAREZ, E. et al. Procyanidins from Grape Pomace are Suitable Inhibitors of Human Endothelial NADPH Oxidase. Journal of Cellular Biochemistry, v.113, p.1386-1396, 2012.
ATKINS, G.B.; JAIN, M.K. Role of Krüppel-Like Transcription Factors in Endothelial Biology. Circulation Research, v.100, p.1686-1695, 2007.

AVELLONE, G. et al. Effects of moderate Sicilian red wine consumption on inflammatory biomarkers of atherosclerosis. European Journal of Clinical Nutrition, v.60, p.41-47, 2006.

AVIRAM, M.; FUHRMAN, B. Wine Flavonoids Protect against LDL Oxidation and Atherosclerosis. Ann. N.Y. Acad. Sci, v.957, p.146-161, 2002.

BAGUL, P.K. et al. Attenuation of insulin resistance, metabolic syndrome and hepatic oxidative stress by resveratrol in fructose-fed rats. Pharmacological Research, v. 66, p. 260- 268, 2012.

BAK, M.; JUN, M.; JEONG, W. Procyanidins from Wild Grape (Vitis amurensis) Seeds Regulate ARE-Mediated Enzyme Expression via Nrf2 Coupled with p38 and PI3K/ Akt Pathway in HepG2 Cells. Int. J. Mol. Sci., v.13, p. 801-818, 2012.

BAUR, J.A.; SINCLAIR, D.A. Therapeutic potential of resveratrol: the in vivo evidence. Nature Reviews, v. 5, p. 493-506, 2006.

BI, X.L. et al. Resveratrol inhibits nitric oxide and TNF-á production by lipopolysaccharide-activated microglia. International Immunopharmacology, v.5, n.1, p.185193, 2005.

BROWN, M.S.; GOLDSTEIN, J.L. Lipoprotein metabolism in the macrophage: implications for cholesterol deposition in atherosclerosis. Annu Rev Biochem, v.52, p. 223-261, 1983.

CIANCIULLI, A. et al. Modulation of NF-jB activation by resveratrol in LPS treated human intestinal cells results in downregulation of PGE2 production and COX-2 expression. Toxicology in Vitro, v. 26, p.1122-1128, 2012.

CHAPPLE, S.J.; SIOW, R.C.M.; MANN, G.E. Crosstalk between Nrf2 and the proteasome: Therapeutic potential of Nrf2 inducers in vascular disease and aging. The International Journal of Biochemistry \& Cell Biology, v. 44, p.1315-1320, 2012.

CHATTERJEE, M. et al. Role of 5-lipoxygenase in resveratrol mediated suppression of 7,12-dimethylbenz(á) anthracene-induced mammary carcinogenesis in rats. European Journal of Pharmacology, v. 668, p.99-106, 2011.

CHUANG, C.; MCINTOSH, M.K. Potential Mechanisms by Which Polyphenol-Rich Grapes Prevent ObesityMediated Inflammation and Metabolic Diseases. The Annual Review of Nutrition, v.31, p.155-76, 2011.

CHUNG, S. et al. Regulation of SIRT1 in cellular functions: role of polyphenols. Archives of Biochemistry and Biophysics, v. 501, p. 79-90, 2010.

CHUNG, E.Y. et al. Resveratrol down-regulates interferonã-inducible inflammatory genes in macrophages: molecular mechanism via decreased STAT-1 activation. Journal of Nutritional Biochemistry, v. 22, p. 902-909, 2011.

Číž, M. et al. The Influence of Wine Polyphenols on Reactive Oxygen and Nitrogen Species Production by Murine Macrophages RAW 264.7. Physiol. Res., v.57, p.393-402, 2008.

CSISZAR, A. et al. Resveratrol attenuates TNF-á induced activation of coronary arterial endothelial cells: role of

Rev. Bras. PI. Med., Campinas, v.15, n.4, p.617-626, 2013. 
NF-kB inhibition. Am. J. Physiol. Heart. Circ. Physiol., v.291, p.1694-1699, 2006.

DEIANA, M. et al. Wine extracts from Sardinian grape varieties attenuate membrane oxidative damage in Caco-2 cell monolayers. Food Chemistry, v.134, p. 2105-2113, 2012.

DEVARAJ, S.; XU, D.Y.; JIALAL, I. C-Reactive Protein Increases Plasminogen Activator Inhibitor-1 Expression and Activity in Human Aortic Endothelial Cells: Implications for the Metabolic Syndrome and Atherothrombosis. Circulation, v.107, p.398-404, 2003.

ESTRUCH, R. et al. A. Different effects of red wine and gin consumption on inflammatory biomarkers of atherosclerosis: a prospective randomized crossover trial. Atherosclerosis, v. 175, p.117-123, 2004.

GRACIA-SANCHO, J. et al. Activation of SIRT1 by resveratrol induces KLF2 expression conferring an endothelial vasoprotective phenotype. Cardiovascular Research, v.85, p.514-519, 2010.

GESSNER, D. K. et al. Inhibition of the pro-inûammatory NF-kB pathway by a grape seed and grape marc meal extract in intestinal epithelial cells. Journal of Animal Physiology and Animal Nutrition, v. 96, p.1074-1083, 2012.

HANSSON, G.K.; LIBBY, P. The immune response in atherosclerosis: a double-edged sword. Nat. Rev. Immunol., v.6, p.508-519, 2006.

HANSSON, G.K.; HERMANSSON, A. The immune system in artherosclerosis. Nature Immunology, v.12, p.204212. 2011.

HAO, H.D.; HE, L.R. Mechanisms of Cardiovascular Protection by Resveratrol. Journal of Medicinal Food, Vol.7. No.3, pp. 290-298, 2004.

KAUR, G. et al. Effect of wine phenolics on cytokineinduced C-reactive protein expression. Journal of Thrombosis and Haemostasis, v.5, p.1309-1317, 2007.

KIM, J.; CHA, Y.N.; SURH, Y.J. A protective role of nuclear factor-erythroid 2-related factor-2 (Nrf2) in inûammatory disorders. Mutat. Res., v. 690, p.12-23, 2010.

KUBOTA, S. et al. Resveratrol Prevents Light-Induced Retinal Degeneration via Suppressing Activator Protein-1 Activation. The American Journal of Pathology, v.177, n.4, 2010.

KUNDU, J.K.; SURH, Y. Molecular basis of chemoprevention by resveratrol: NF-kB and AP-1 as potential targets. Mutation Research, v.555, p.65-80, 2004.

LANÇON, A. et al. Control of MicroRNA Expression as a New Way for Resveratrol to Deliver Its Beneûcial Effects. J. Agric. Food Chem., v. 60, p.8783"8789, 2012.

LASTRA, C.A.; VILLEGAS, I. Resveratrol as an antioxidant and pro-oxidant agent: mechanisms and clinical implications. Biochemical Society Transactions, v.35, n.5, p.1156-1160, 2007.

LEE, J.H. et al. Dietary phytochemicals and cancer prevention: Nrf2 signaling, epigenetics, and cell death mechanisms in blocking cancer initiation and progression. Pharmacology \& Therapeutics, p. 1-19, 2012.

LEIFERT, W.R.; ABEYWARDENA, M.Y. Grape seed and red wine polyphenol extracts inhibit cellular cholesterol uptake, cell proliferation, and 5-lipoxygenase activity. Nutrition Research, v.28, p.842-850, 2008.
LEIRO, A.J. et al. Effect of cis-resveratrol on genes involved in nuclear factor kappa B signaling. International Immunopharmacology, v.5, p.393-406, 2005.

LIBBY, P.; RIDKER, P.M.; MASERI, A. Inflammation and Atherosclerosis. Circulation, v.105, p.1135-1143, 2002.

LUZ, P.L.; COIMBRA, S.R. Wine, alcohol and atherosclerosis: clinical evidences and mechanisms. Brazilian Journal of Medical and Biological Research, v.37, p.1275-1295, 2004.

MAGGI-CAPEYRON, M. et al. Wine Phenolic Antioxidants Inhibit AP-1 Transcriptional Activity. J. Agric. Food Chem., v49, p.5646-5652, 2001.

MANACH, C.; MAZUR, A.; SCALBERT, A. Polyphenols and prevention of cardiovascular diseases. Curr. Opin. Lipidol., v.16, p.77-84, 2005.

MARTINDALE, J.L.; HOLBROOK, N.J. Cellular response to oxidative stress: sgnaling for suicide and survival. Journal of Celluar Physiology, v.192, p.1-15, 2002.

MCLAREN, J.E. et al. The TNF-Like Protein 1A-Death Receptor 3 pathway promotes macrophage foam cell formation in vitro. The Journal of Immunology, v.184, p. 5827-5834, 2010.

MEHTA, J.L. et al. Lectin-like, oxidized low-density lipoprotein receptor-1 (LOX-1): A critical player in the development of atherosclerosis and related disorders. Cardiovascular Research, v.69, p. 36 - 45, 2006.

MOVAHED, A. et al. Resveratrol protects adult cardiomyocytes against oxidative stress mediated cell injury. Archives of Biochemistry and Biophysics, v.527, n. 2, p. 74-80, 2012.

NDIAYE, M. et al. Red wine polyphenols cause endothelium-dependent EDHF-mediated relaxations in porcine coronary arteries via a redox-sensitive mechanism. Biochemical and Biophysical Research Communications, v.310, p.371-377, 2003.

NOGUER, M.A.; CEREZO, A.B.; NAVARRO, E.D.; GARCIA-PARRILLA, M.C. Intake of alcohol-free red wine modulates antioxidant enzyme activities in a human intervention study. Pharmacological Research, v. 65, p.609- 614, 2012.

NORATTO, G.D. et al. Polyphenolics from Açaí (Euterpe oleracea Mart.) and Red Muscadine Grape (Vitis rotundifolia) Protect Human Umbilical Vascular Endothelial Cells (HUVEC) from Glucose- and Lipopolysaccharide (LPS)-Induced Inflammation and Target MicroRNA-126. J. Agric. Food Chem., v. 59, p.7999-8012, 2011

OAK, M.; BEDOUIA, J.; SCHINI-KERTH, V.B. Antiangiogenic properties of natural polyphenols from red wine and green tea. Journal of Nutritional Biochemistry, v.16, p.1-8, 2005.

OVERMAN, A. et al. Polyphenol-rich grape powder extract (GPE) attenuates inflammation in human macrophages and in human adipocytes exposed to macrophageconditioned media. International Journal of Obesity, v.34, p.800-808, 2010.

PALSAMY, P.; SUBRAMANIAN, S. Resveratrol protects diabetic kidney by attenuating hyperglycemia-mediated oxidative stress and renal inûammatory cytokines via Nrf2-Keap1 signaling. Biochimica et Biophysica Acta, v.1812, p. 719-731, 2011.

RENAUD, S.; LORGERIL, M. Wine, alcohol, platelets, and the French paradox for coronary heart disease. Lancet,

Rev. Bras. PI. Med., Campinas, v.15, n.4, p.617-626, 2013. 
v.339, p.1523-1526, 1992.

RAHMAN, I. Dietary polyphenols mediated regulation of oxidative stress and chromatin remodeling in inflammation. Nutrition Reviews, v.66, n.1, p.4245, 2008.

ROBBESYN, F.; SALVAYRE, R.; NEGRE-SALVAYRE, A. Dual Role of Oxidized LDL on the NF-KappaB Signaling Pathway. Free Radical Research, v.38, n.6, p.541-55, 2004.

ROCHA, V.Z.; LIBBY, P. Obesity, inflammation and atherosclerosis. Nat. Rev. Cardiol., v.6, 6, p.399409, 2009

RODRIGO, R.; MIRANDA, A.; VERGARA, L. Modulation of endogenous antioxidant system by wine polyphenols in human disease. Clinica Chimica Acta, v. 412, p. 410-424, 2011.

RODRÍGUEZ-RAMIRO, I. et al. Procyanidin B2 induces Nrf2 translocation and glutathione S-transferase P1 expression via ERKs and p38-MAPK pathways and protect human colonic cells against oxidative stress. Eur J Nutr., v.51, p. 881-892, 2012.

SACANELLA, E. et al. Down-regulation of adhesion molecules and other inflammatory biomarkers after moderate wine consumption in healthy women: a randomized trial. American Journal of Clinical Nutrition, v.86, n.5, p.1463-1469, 2007.

$\mathrm{SHAO}$, B. et al. Proteomics analysis of human umbilical vein endothelial cells treated with resveratrol. Amino Acids, v.43, p. 1671-1678, 2012.

SOYLEMEZ, S. et al. The effect of long-term resveratrol treatment on relaxation to estrogen in aortae from male and female rats: role of nitric oxide and superoxide. Vasc. Pharmacol., v.49, p.97-105, 2008.

SOYLEMEZ, S.; SEPICI, A.; AKAR F. Resveratrol Supplementation Gender Independently Improves Endothelial Reactivity and Suppresses Superoxide Production in Healthy Rats. Cardiovasc. Drugs Ther., v.23, p.449-458, 2009.

STEINBERG, D. et al. Lipoproteins and the pathogenesis of atherosclerosis. Circulation, v.80, p. 719-723, 1989.

STEINBERG, D.; WITZTUM, J.L. Oxidized low-density lipoprotein and atherosclerosis. Arterioscler. Thromb. Vasc. Biol., v.30, p.2311-2316, 2010.

SUH, J. et al. Polyphenols prevent lipid abnormalities and arterial dysfunction in hamsters on a high-fat diet: a comparative study of red grape and white persimmon wines. Food Funct., v.2, p. 555-56, 2011.

TAKAYA, K. et al. Validation of the multiple sensor mechanism of the Keap1-Nrf2 system. Free Radical
Biology and Medicine, v. 53, p. 817-827, 2012.

TEDGUI, A.; MALLAT, Z. Cytokines in Atherosclerosis: Pathogenic and Regulatory Pathways. Physiol. Rev., v.86, p.515-581, 2006.

TILI, E. et al. Resveratrol decreases the levels of miR155 by upregulating miR-663, a new anti-inûammatory microRNA targeting JunB and JunD. Carcinogenesis, V.31, p.1561"1566, 2010.

UNGVARI, Z. et al. Resveratrol confers endothelial protection via activation of the antioxidant transcription factor Nrf2. Am J Physiol Heart Circ Physiol., v.299, n.1, p.18-24, 2010.

URPÍ-SARDÀ, M. et al. Uptake of Diet Resveratrol into the Human Low-Density Lipoprotein. Identification and Quantification of Resveratrol Metabolites by Liquid Chromatography Coupled with Tandem Mass Spectrometry. Anal. Chem., v.77, p.3149-3155, 2005.

VITA, J.A. Polyphenols and cardiovascular disease: effects on endothelial and platelet function. Am. J. Clin. Nutr., v. 81, p.292-297, 2005.

WARBOYS, C.M. et al. The role of blood flow in determining the sites of atherosclerotic plaques. Medicine Reports, v.3, n.5, p.1-8, 2011.

XIA, E. et al. Biological Activities of Polyphenols from Grapes. Int. J. Mol. Sci., v.11, p.622-646, 2010. Xu, Q.; Si, L. Resveratrol role in cardiovascular and metabolic health and potential mechanisms of action. Nutrition Research, v. 32, p. 648 - 658, 2012.

YAO, H.; RAHMAN, I. Perspectives on translational and therapeutic aspects of SIRT1 in inûammaging and senescence. Biochemical Pharmacology, v.84, p. 1332-1339, 2012.

YI, C. et al. Resveratrol activates AMPK and suppresses LPS-induced NF-kB-dependent COX-2 activation in RAW 264.7 macrophage cells. Anat Cell Biol., v.44, p.194-203, 2011.

YUN, J. et al. Resveratrol up-regulates SIRT1 and inhibits cellular oxidative stress in the diabetic milieu: mechanistic insights. Journal of Nutritional Biochemistry, v.23, p.699-705, 2012.

ZERN, T.L. et al. Grape Polyphenols Exert a Cardioprotective Effect in Pre- and Postmenopausal Women by Lowering Plasma Lipids and Reducing Oxidative Stress. The Journal of Nutrition, v.135, p.1911-1917, 2005.

ZHANG, $\mathrm{H}$. et al. Resveratrol meliorates ionizing irradiation-induced long-term hematopoietic stem cell injury in mice. Free Radical Biology and Medicine, v.54, p.40-50, 2013. 